\title{
EXISTENCE OF WEAK-RENORMALIZED SOLUTION FOR A NONLINEAR SYSTEM
}

\author{
B. CLIMENT
}

\begin{abstract}
We prove an existence result for a coupled system of the reactiondiffusion kind. The fact that no growth condition is assumed on some nonlinear terms motivates the search of a weak-renormalized solution.
\end{abstract}

\section{Introduction. Description of the problem}

This paper investigates the existence of a solution for the nonlinear system

$$
\left\{\begin{array}{cc}
-\Delta u-\nabla \cdot\left(\beta(v) X^{\prime}(u)\right)=f & \text { in } \Omega, \\
-\Delta v-\nabla \cdot\left(\beta^{\prime}(v) X(u)\right)=g & \text { in } \Omega, \\
u=0, \quad v=0 \quad \text { on } \partial \Omega, &
\end{array}\right.
$$

where $\Omega$ denotes a bounded open subset of $\mathbb{R}^{N}, X$ is a $C^{1}$ bounded $\mathbb{R}^{N}$-valued function on $\mathbb{R}$, i.e.

$$
X \in\left(C^{1}(\mathbb{R})\right)^{N} \cap\left(C_{b}^{0}(\mathbb{R})\right)^{N},
$$

$\beta$ is a function whose second derivatives are bounded, i.e.

$$
\beta \in W^{2, \infty}(\mathbb{R})
$$

and

$$
f, g \in H^{-1}(\Omega) .
$$

Here, the main difficulty to find a solution is that no growth restrictions are assumed on $X^{\prime}$. Since $f$ and $g$ belong to $H^{-1}(\Omega)$, it is natural to look for solutions $u$ and $v$ belonging to $H_{0}^{1}(\Omega)$. Thus, it is not clear how 2000 Mathematics Subject Classification: 35D05, 35J65.

Servicio de Publicaciones. Universidad Complutense. Madrid, 2002 
to give a sense to $\nabla \cdot\left(\beta(v) X^{\prime}(u)\right)$. This inconvenient can be overcome by introducing a weak-renormalized formulation of this problem, essentially obtained through pointwise multiplication of the first equation of (1) by $h(u)$, where $h$ belongs to $C_{0}^{1}(\mathbb{R})$, that is, $h \in C^{1}(\mathbb{R})$ and its support is compact.

Remark. We can view this system as a simplified model of a nonlinear elasticity problem characterized by a constitutive law of the form

$$
\sigma=\sigma_{l}+Y(u)
$$

where

$$
\left(\sigma_{l}\right)_{i j}=\sum a_{i j k l} \varepsilon_{k l}(u), \quad \varepsilon_{k l}(u)=\frac{1}{2}\left(\frac{\partial u_{k}}{\partial x_{l}}+\frac{\partial u_{l}}{\partial x_{k}}\right), \quad Y_{i j} \in C^{0}\left(\mathbb{R}^{2}\right) .
$$

Indeed, the conservation of momentum reads

$$
\nabla \cdot \sigma=F
$$

( $F$ is given), which is in some sense a generalization of (1). In this paper, we study the case in which

$$
Y(u)=\left(\begin{array}{ll}
\beta\left(u_{2}\right) X_{1}^{\prime}\left(u_{1}\right) & \beta^{\prime}\left(u_{2}\right) X_{1}\left(u_{1}\right) \\
\beta\left(u_{2}\right) X_{2}^{\prime}\left(u_{1}\right) & \beta^{\prime}\left(u_{2}\right) X_{2}\left(u_{1}\right)
\end{array}\right)
$$

\section{The main result}

Theorem 2.1. Under the assumptions (2), (3), (4), there exists $\{u, v\}$, with $u, v \in H_{0}^{1}(\Omega)$, such that the second equation in (1) is satisfied in the usual weak or distributional sense and the first equation holds in the following sense:

$$
\left\{\begin{aligned}
-\nabla \cdot & (h(u) \nabla u)+\nabla u \cdot \nabla h(u)-\nabla \cdot\left(\beta(v) h(u) X^{\prime}(u)\right) \\
& +\beta(v) X^{\prime}(u) \cdot \nabla h(u)=f h(u) \text { in } \mathcal{D}^{\prime}(\Omega) \quad \forall h \in C_{0}^{1}(\mathbb{R}) .
\end{aligned}\right.
$$

A couple $\{u, v\}$ as above will be called a weak-renormalized solution to (1). 
Remark. In (5), every term belongs to $\mathcal{D}^{\prime}(\Omega)$. Indeed, $h(u)$ belongs to $H_{0}^{1}(\Omega)$, the first term is in $H^{-1}(\Omega)$. The second one is in $L^{1}(\Omega)$. For instance, since $h$ has a compact support, we can put

$$
h(u) X^{\prime}(u)=h(u) X^{\prime}\left(T_{M}(u)\right) \quad \text { and } \quad h^{\prime}(u) X^{\prime}(u)=h^{\prime}(u) X^{\prime}\left(T_{M}(u)\right)
$$

for some $M>0$, where $T_{M}$ is the usual truncation at level $M$. Thus, we see that the third term in the left belongs to $W^{-1, \infty}(\Omega)$ and the fourth term belongs to $L^{2}(\Omega)$.

Remark. Renormalized solutions to PDE's were introduced by R. DiPerna and P.L. Lions in [4] in the framework of the Boltzmann equation. They have been used in connection with various nonlinear elliptic equations by P. Benilan et al. [2], L. Boccardo et al. [3] and P.L. Lions and F. Murat [6] (see also [7]). In the analysis of existence results for systems, weak-renormalized solutions were first considered by R. Lewandowski [5] (see also [1]).

In this paper, in order to solve (1), we will extend the techniques used in [3] in the context of a single equation.

Remark. With regard to uniqueness, it is an open problem. If we follow the classical argument of considering two solutions $u^{i}, v^{i}$ for $i=1,2$ of (1), and we compute the difference of (5) written for $u^{1}, v^{1}$ and for $u^{2}, v^{2}$, we find expressions with terms of the form $X^{\prime}(\cdot) u$ that we are not able to estimate. There is another argument, due to P. L. Lions and F. Murat [7], which leads to the uniqueness of renormalized solutions, but it cannot be applied here.

\section{The proof of theorem 2.1}

First step. The introduction of a family of approximations.

For each $\varepsilon>0$, let us put $X^{\varepsilon}(s)=X\left(T_{1 / \varepsilon}(s)\right)$ for all $s \in \mathbb{R}$. We will introduce the following approximation to $(1)$ :

$$
\left\{\begin{array}{c}
-\Delta u^{\varepsilon}-\nabla \cdot\left(\beta\left(v^{\varepsilon}\right)\left(X^{\varepsilon}\right)^{\prime}\left(u^{\varepsilon}\right)\right)=f \quad \text { in } \Omega, \\
-\Delta v^{\varepsilon}-\nabla \cdot\left(\beta^{\prime}\left(v^{\varepsilon}\right) X\left(u^{\varepsilon}\right)\right)=g \quad \text { in } \Omega, \\
u^{\varepsilon}, v^{\varepsilon} \in H_{0}^{1}(\Omega),
\end{array}\right.
$$


In order to solve (6), we will apply Schauder's theorem. Thus, for any given $\varepsilon$ and $\{u, v\} \in L^{2}(\Omega) \times L^{2}(\Omega)$, we set $R^{\varepsilon}(\{u, v\})=\left\{u^{\varepsilon}, v^{\varepsilon}\right\}$, with $\left\{u^{\varepsilon}, v^{\varepsilon}\right\}$ being the unique solution to the linear system

$$
\left\{\begin{aligned}
-\Delta u^{\varepsilon}=f+\nabla \cdot\left(\beta(v)\left(X^{\varepsilon}\right)^{\prime}(u)\right) & \text { in } \Omega, \\
-\Delta v^{\varepsilon}=g+\nabla \cdot\left(\beta^{\prime}(v) X(u)\right) & \text { in } \Omega, \\
u^{\varepsilon}, v^{\varepsilon} \in H_{0}^{1}(\Omega), &
\end{aligned}\right.
$$

Obviously, $R^{\varepsilon}=R_{3} \circ R_{2} \circ R_{1}^{\varepsilon}$, where

- $R_{1}^{\varepsilon}: L^{2}(\Omega) \times L^{2}(\Omega) \mapsto H^{-1}(\Omega) \times H^{-1}(\Omega)$ is the nonlinear continuous mapping given by

$$
\left\{\begin{array}{c}
R_{1}^{\varepsilon}(\{u, v\})=\left\{f+\nabla \cdot\left(\beta(v)\left(X^{\varepsilon}\right)^{\prime}(u)\right), g+\nabla \cdot\left(\beta^{\prime}(v) X(u)\right)\right\} \\
\forall\{u, v\} \in L^{2}(\Omega) \times L^{2}(\Omega),
\end{array}\right.
$$

- $R_{2}: H^{-1}(\Omega) \times H^{-1}(\Omega) \mapsto H_{0}^{1}(\Omega) \times H_{0}^{1}(\Omega)$ associates to each $\{f, g\} \in H^{-1}(\Omega) \times H^{-1}(\Omega)$ the unique solution $\{w, z\}$ of the following linear system

$$
\left\{\begin{array}{c}
-\Delta w=f \quad \text { in } \Omega, \\
-\Delta z=g \quad \text { in } \Omega \\
w, z \in H_{0}^{1}(\Omega),
\end{array}\right.
$$

- $R_{3}$ is the compact embedding of $H_{0}^{1}(\Omega) \times H_{0}^{1}(\Omega)$ into $L^{2}(\Omega) \times L^{2}(\Omega)$.

Since $R_{1}^{\varepsilon}$ maps the whole space $L^{2}(\Omega) \times L^{2}(\Omega)$ inside a ball, Schauder's theorem can be applied and (6) possesses at least one solution $\left\{u^{\varepsilon}, v^{\varepsilon}\right\}$.

Second step. A priori estimates and weak convergence.

Choosing $u^{\varepsilon}$ and $v^{\varepsilon}$ as test functions in the first and second equation in (6) respectively, one finds:

$$
\begin{gathered}
\int_{\Omega} \nabla u^{\varepsilon} \nabla u^{\varepsilon}+\int_{\Omega} \beta\left(v^{\varepsilon}\right)\left(X^{\varepsilon}\right)^{\prime}\left(u^{\varepsilon}\right) \cdot \nabla u^{\varepsilon}=\left\langle f, u^{\varepsilon}\right\rangle_{H^{-1}, H_{0}^{1}} . \\
\int_{\Omega} \nabla v^{\varepsilon} \nabla v^{\varepsilon}+\int_{\Omega} \beta^{\prime}\left(v^{\varepsilon}\right) X\left(u^{\varepsilon}\right) \cdot \nabla v^{\varepsilon}=\left\langle g, v^{\varepsilon}\right\rangle_{H^{-1}, H_{0}^{1}} .
\end{gathered}
$$


For $\varepsilon$ sufficiently small, $X=X \circ T_{1 / \varepsilon}=X^{\varepsilon}$, whence we can replace $X\left(u^{\varepsilon}\right)$ by $X^{\varepsilon}\left(u^{\varepsilon}\right)$ in $(9)$.

Let us introduce the function $H=\left(H_{1}, H_{2}, \ldots, H_{n}\right)$, with

$$
H_{i}(t, s)=\int_{0}^{s} \beta(0)\left(X_{i}^{\varepsilon}\right)^{\prime}(\theta) d \theta+\int_{0}^{t} \beta^{\prime}(\theta) X_{i}^{\varepsilon}(s) d \theta
$$

Then,

$$
\int_{\Omega} \beta\left(v^{\varepsilon}\right)\left(X_{\varepsilon}\right)^{\prime}\left(u^{\varepsilon}\right) \cdot \nabla u^{\varepsilon}+\int_{\Omega} \beta^{\prime}\left(v^{\varepsilon}\right) X_{\varepsilon}\left(u^{\varepsilon}\right) \cdot \nabla v^{\varepsilon}=\int_{\Omega} \nabla \cdot H\left(u^{\varepsilon}, v^{\varepsilon}\right)=0
$$

thanks to Stokes' theorem. Summing (8) and (9), we obtain

$$
\int_{\Omega}\left|\nabla u^{\varepsilon}\right|^{2}+\int_{\Omega}\left|\nabla v^{\varepsilon}\right|^{2}=\left\langle f, u^{\varepsilon}\right\rangle_{H^{-1}, H_{0}^{1}}+\left\langle g, v^{\varepsilon}\right\rangle_{H^{-1}, H_{0}^{1}}
$$

and

$$
\left\|u^{\varepsilon}\right\|_{H_{0}^{1}}^{2}+\left\|v^{\varepsilon}\right\|_{H_{0}^{1}}^{2} \leq\|f\|_{H^{-1}}^{2}+\|g\|_{H^{-1}}^{2} .
$$

Consequently, at least for a subsequence, still indexed by $\varepsilon$, we can conclude that

$$
\begin{aligned}
& u^{\varepsilon} \rightarrow u, v^{\varepsilon} \rightarrow v \quad \text { weakly in } H_{0}^{1}(\Omega), \\
& u^{\varepsilon} \rightarrow u, v^{\varepsilon} \rightarrow v \quad \text { strongly in } L^{p}(\Omega) \quad \forall p \in\left[1,2^{\star}\right) \text { and a.e. }
\end{aligned}
$$

Here, we have denoted by $2^{\star}$ the exponent furnished by the Sobolev embedding theorem, that is

$$
\left\{\begin{array}{l}
2^{\star}=\frac{2 N}{N-2} \quad \text { if } N \geq 3, \\
2^{\star}<+\infty \text { arbitrarily large if } N=2 .
\end{array}\right.
$$

Third step. The strong convergence of $v^{\varepsilon}$ in $H_{0}^{1}$.

It is easy to see that $v$ is a weak solution to the problem

$$
\left\{\begin{array}{c}
-\Delta v-\nabla \cdot\left(\beta^{\prime}(v) X(u)\right)=g \text { in } \Omega, \\
v=0 \quad \text { on } \partial \Omega
\end{array}\right.
$$

Indeed, since $\beta^{\prime}$ and $X$ are continuous and bounded, it is clear that $\beta^{\prime}\left(v^{\varepsilon}\right) \rightarrow \beta^{\prime}(v)$ strongly in $L^{p}$ for all $p \in\left[1,2^{\star}\right)$ and $X\left(u^{\varepsilon}\right) \rightarrow X(u)$ 
strongly in $L^{r}$ for all $r \in[1,+\infty)$. This enables us to pass to the limit in the second equation in (6).

From (11), we also see that

$$
\int_{\Omega}|\nabla v|^{2}=-\int_{\Omega} \beta^{\prime}(v) X(u) \cdot \nabla v+\int_{\Omega} g v .
$$

Let us use $v^{\varepsilon}$ as a test function in the second equation in (6). We find:

$$
\int_{\Omega}\left|\nabla v^{\varepsilon}\right|^{2}=-\int_{\Omega} \beta^{\prime}\left(v^{\varepsilon}\right) X\left(u^{\varepsilon}\right) \cdot \nabla v^{\varepsilon}+\int_{\Omega} g v^{\varepsilon} .
$$

Arguing as above, we can pass to the limit in the right hand side in (13). Accordingly, we have:

$$
\int_{\Omega}\left|\nabla v^{\varepsilon}\right|^{2} \rightarrow-\int_{\Omega} \beta^{\prime}(v) X(u) \cdot \nabla v+\int_{\Omega} g v .
$$

This, combined with (12), gives the convergence in norm in $H_{0}^{1}$ for $v^{\varepsilon}$ and, consequently,

$$
v^{\varepsilon} \rightarrow v \quad \text { strongly in } H_{0}^{1} \text {. }
$$

Fourth step. The strong convergence of $u^{\varepsilon}$ in $H_{0}^{1}$.

We will first prove that

$$
\lim _{K \rightarrow+\infty}\left(\limsup _{\varepsilon \rightarrow 0} \int_{\left\{\left|u^{\varepsilon}\right|>K\right\}}\left|\nabla u^{\varepsilon}\right|^{2}\right)=0
$$

Thus, let us consider the test functions $u^{\varepsilon}-T_{K}\left(u^{\varepsilon}\right)$ in the first equation in (6). Notice that

$$
\nabla\left(u^{\varepsilon}-T_{K}\left(u^{\varepsilon}\right)\right)= \begin{cases}\nabla u^{\varepsilon} & \text { if }\left|u^{\varepsilon}\right| \geq K, \\ 0 & \text { if }\left|u^{\varepsilon}\right|<K .\end{cases}
$$

Hence,

$$
\begin{gathered}
\int_{\left\{\left|u^{\varepsilon}\right| \geq K\right\}}\left|\nabla u^{\varepsilon}\right|^{2}+\int_{\Omega} \beta\left(v^{\varepsilon}\right)\left(1-T_{K}^{\prime}\left(u^{\varepsilon}\right)\right)\left(X^{\varepsilon}\right)^{\prime}\left(u^{\varepsilon}\right) \cdot \nabla u^{\varepsilon} \\
=\left\langle f, u^{\varepsilon}-T_{K}\left(u^{\varepsilon}\right)\right\rangle .
\end{gathered}
$$


We can put $\left(1-T_{K}^{\prime}\left(u^{\varepsilon}\right)\right)\left(X^{\varepsilon}\right)^{\prime}\left(u^{\varepsilon}\right) \cdot \nabla u^{\varepsilon}=\nabla \cdot Y_{K}^{\varepsilon}\left(u^{\varepsilon}\right)$, where

$$
\left(Y_{K}^{\varepsilon}\right)_{i}(t)=\int_{0}^{t}\left(1-T_{K}^{\prime}(\theta)\right)\left(X^{\varepsilon}\right)^{\prime}(\theta) d \theta .
$$

Thus, the second term in the left hand side of (16) can be written in the form

$$
\int_{\Omega}\left(\nabla \cdot Y_{K}^{\varepsilon}\left(u^{\varepsilon}\right)\right) \beta\left(v^{\varepsilon}\right)=-\int_{\Omega} Y_{K}^{\varepsilon}\left(u^{\varepsilon}\right) \cdot \nabla \beta\left(v^{\varepsilon}\right) .
$$

Moreover,

$$
Y_{K}^{\varepsilon}(s)= \begin{cases}X^{\varepsilon}(s)-X^{\varepsilon}(K) & \text { if } s>K, \\ 0 & \text { if }\left|u^{\varepsilon}\right| \leq K, \\ X^{\varepsilon}(s)-X^{\varepsilon}(-K) & \text { if } s<-K .\end{cases}
$$

Since $X \in C_{b}^{0}(\mathbb{R})^{N}$, for $\varepsilon>0$ sufficiently small, $Y_{K}^{\varepsilon}$ is independent of $\varepsilon$ and $Y_{K}^{\varepsilon}\left(u^{\varepsilon}\right)$ is bounded by a constant independent of $\varepsilon$. We also have

$$
\limsup _{\varepsilon \rightarrow 0}\left|Y_{K}^{\varepsilon}\left(u^{\varepsilon}\right)\right| \leq|X(u)-X(K)| \mathbb{1}_{\{u>K\}}+|X(u)-X(-K)| \mathbb{1}_{\{u<-K\}}
$$

for all $K>0$. Therefore,

$$
\left\{\begin{array}{c}
\limsup _{\varepsilon \rightarrow 0} \int_{\left\{\left|u^{\varepsilon}\right|>K\right\}}\left|\nabla u^{\varepsilon}\right|^{2} \leq \int_{\Omega}|X(u)-X(K)| \cdot|\nabla \beta(v)| \mathbb{1}_{\{u>K\}} \\
\quad+\int_{\Omega}|X(u)-X(-K)| \cdot|\nabla \beta(v)| \mathbb{1}_{\{u<-K\}}+\left\langle f, u-T_{K}(u)\right\rangle,
\end{array}\right.
$$

whence

$$
\left\{\begin{array}{l}
\lim _{K \rightarrow+\infty}\left(\limsup _{\varepsilon \rightarrow 0} \int_{\left\{\left|u^{\varepsilon}\right|>K\right\}}\left|\nabla u^{\varepsilon}\right|^{2}\right) \\
\leq \lim _{K \rightarrow+\infty}\left[\int_{\Omega}|X(u)-X(K)| \cdot|\nabla \beta(v)| \mathbb{1}_{\{u>K\}}\right. \\
\left.\quad+\int_{\Omega}|X(u)-X(-K)| \cdot|\nabla \beta(v)| \mathbb{1}_{\{u<-K\}}\right] \\
\quad+\lim _{K \rightarrow+\infty}\left\langle f, u-T_{K}(u)\right\rangle=0 .
\end{array}\right.
$$


This proves (15). Let us introduce the sets $F_{i, j}^{\varepsilon}$,

$$
F_{i, j}^{\varepsilon}=\left\{x \in \Omega:\left|u^{\varepsilon}-T_{j}(u)\right| \leq i\right\} .
$$

We are now going to prove that

$$
\lim _{j \rightarrow+\infty}\left(\limsup _{\varepsilon \rightarrow 0} \int_{F_{i, j}^{\varepsilon}}\left|\nabla\left(u^{\varepsilon}-T_{j}(u)\right)\right|^{2}\right)=0 \quad \forall i \geq 1 .
$$

Thus, let us use $T_{i}\left(u^{\varepsilon}-T_{j}(u)\right)$ as test function in the first equation of (6). We obtain

$$
\begin{gathered}
\int_{\Omega} \nabla u^{\varepsilon} \cdot \nabla T_{i}\left(u^{\varepsilon}-T_{j}(u)\right)+\int_{\Omega} \beta\left(v^{\varepsilon}\right)\left(X^{\varepsilon}\right)^{\prime}\left(u^{\varepsilon}\right) \cdot \nabla T_{i}\left(u^{\varepsilon}-T_{j}(u)\right) \\
=\left\langle f, T_{i}\left(u^{\varepsilon}-T_{j}(u)\right)\right\rangle .
\end{gathered}
$$

Let us notice that

$$
\nabla T_{i}\left(u^{\varepsilon}-T_{j}(u)\right)=0 \text { in } \Omega \backslash F_{i, j}^{\varepsilon} .
$$

We can then write (20) in the form

$$
\begin{gathered}
\int_{F_{i, j}^{\varepsilon}} \nabla u^{\varepsilon} \cdot \nabla T_{i}\left(u^{\varepsilon}-T_{j}(u)\right)+\int_{F_{i, j}^{\varepsilon}} \beta\left(v^{\varepsilon}\right)\left(X^{\varepsilon}\right)^{\prime}\left(u^{\varepsilon}\right) \cdot \nabla T_{i}\left(u^{\varepsilon}-T_{j}(u)\right) \\
=\left\langle f, T_{i}\left(u^{\varepsilon}-T_{j}(u)\right)\right\rangle .
\end{gathered}
$$

Since

$$
\left|u^{\varepsilon}\right| \leq\left|u^{\varepsilon}-T_{j}(u)\right|+\left|T_{j}(u)\right| \leq i+j \quad \text { if } x \in F_{i, j}^{\varepsilon},
$$

we can write $T_{1 / \varepsilon}\left(u^{\varepsilon}\right)=T_{i+j}\left(u^{\varepsilon}\right)$ for all $x \in F_{i, j}^{\varepsilon}$ whenever $\varepsilon$ is sufficiently small. This gives:

$$
\left(X^{\varepsilon}\right)^{\prime}\left(u^{\varepsilon}\right)=X^{\prime}\left(T_{i+j}\left(u^{\varepsilon}\right)\right) T_{i+j}^{\prime}\left(u^{\varepsilon}\right)=X^{\prime}\left(T_{i+j}\left(u^{\varepsilon}\right)\right) \text { in } F_{i, j}^{\varepsilon} .
$$

Thus, for small $\varepsilon>0$, the second term in the left in (21) is

$$
\int_{F_{i, j}^{\varepsilon}} \beta\left(v^{\varepsilon}\right) X^{\prime}\left(T_{i+j}\left(u^{\varepsilon}\right)\right) \cdot \nabla T_{i}\left(u^{\varepsilon}-T_{j}(u)\right)
$$

and converges to

$$
\int_{\Omega} \beta(v) X^{\prime}\left(T_{i+j}(u)\right) \cdot \nabla T_{i}\left(u-T_{j}(u)\right)
$$


as $\varepsilon \rightarrow 0$, since

$$
T_{i}\left(u^{\varepsilon}-T_{j}(u)\right) \rightarrow T_{i}\left(u-T_{j}(u)\right) \text { weakly in } H_{0}^{1}
$$

and $\beta\left(v^{\varepsilon}\right) X^{\prime}\left(T_{i+j}\left(u^{\varepsilon}\right)\right)$ is bounded in $\left(L^{\infty}(\Omega)\right)^{N}$ and converges a.e. to $\beta(v) X^{\prime}\left(T_{i+j}(u)\right)$.

Let us introduce $H^{i, j}=\left(H_{1}^{i, j}, H_{2}^{i, j}, \ldots, H_{N}^{i, j}\right)$, with

$$
H^{i, j}(s)=\int_{0}^{s} T_{i}^{\prime}\left(\theta-T_{j}(\theta)\right)\left(1-T_{j}^{\prime}(\theta)\right) X^{\prime}\left(T_{i+j}(\theta)\right) d \theta .
$$

Then (22) can be rewritten in the form

$$
\int_{\Omega}\left(\nabla \cdot H_{K}^{i, j}(u)\right) \beta(v)=-\int_{\Omega} H^{i, j}(u) \cdot \nabla \beta(v)
$$

Moreover, it is not difficult to check that

$$
H^{i, j}(u)= \begin{cases}X(i+j)-X(j) & \text { if } j<|u|<i+j, \\ 0 & \text { otherwise. }\end{cases}
$$

For any $i$, we have $H^{i, j}(u) \rightarrow 0$ a. e. as $j \rightarrow+\infty$. Since $\mathrm{X}$ is bounded, $H^{i, j}(u)$ is also bounded. Thus, we obtain from Lebesgue's theorem that

$$
\int_{\Omega} H^{i, j}(u) \cdot \nabla \beta(v) \rightarrow 0 \quad \text { as } j \rightarrow \infty
$$

for all $i \geq 1$. Recalling (20) we see we have proved the following:

$$
\lim _{j \rightarrow+\infty}\left(\lim _{\varepsilon \rightarrow 0} \int_{F_{i, j}^{\varepsilon}} \nabla u^{\varepsilon} \cdot \nabla T_{i}\left(u^{\varepsilon}-T_{j}(u)\right)\right)=\lim _{j \rightarrow+\infty}\left\langle f, T_{i}\left(u-T_{j}(u)\right)\right\rangle .
$$

On the other hand,

$$
\begin{gathered}
\lim _{j \rightarrow+\infty}\left(\lim _{\varepsilon \rightarrow 0} \int_{F_{i, j}^{\varepsilon}} \nabla T_{j}(u) \cdot \nabla T_{i}\left(u^{\varepsilon}-T_{j}(u)\right)\right) \\
=\lim _{j \rightarrow+\infty} \int_{\Omega} \nabla T_{j}(u) \cdot \nabla T_{i}\left(u-T_{j}(u)\right) .
\end{gathered}
$$


Consequently,

$$
\begin{gathered}
\lim _{j \rightarrow+\infty}\left(\lim _{\varepsilon \rightarrow 0} \int_{F_{i, j}^{\varepsilon}}\left|\nabla\left(u^{\varepsilon}-T_{j}(u)\right)\right|^{2}\right) \\
=\lim _{j \rightarrow+\infty}\left(\left\langle f, T_{i}\left(u-T_{j}(u)\right)\right\rangle-\int_{\Omega} \nabla T_{j}(u) \cdot \nabla T_{i}\left(u-T_{j}(u)\right)\right) .
\end{gathered}
$$

Notice that, the terms on the right hand side of (24) can be bounded as follows:

$$
\begin{gathered}
\left\langle f, T_{i}\left(u-T_{j}(u)\right)\right\rangle-\int_{\Omega} \nabla T_{j}(u) \cdot \nabla T_{i}\left(u-T_{j}(u)\right) \\
\leq\left(\|f\|_{H^{-1}}+\|u\|\right)\left\|u-T_{j}(u)\right\|
\end{gathered}
$$

and this converges to 0 as $j \rightarrow+\infty$. Therefore, (19) is satisfied.

We can now prove that $u^{\varepsilon}$ converges strongly in $H_{0}^{1}$. Indeed, obseve that, if $x \in \Omega \backslash F_{i, j}^{\varepsilon}$, then

$$
\left|u^{\varepsilon}\right| \geq\left|u^{\varepsilon}-T_{j}(u)\right|-\left|T_{j}(u)\right| \geq i-j,
$$

so that $\Omega \backslash F_{i, j}^{\varepsilon} \subset E_{i-j}^{\varepsilon}$, with

$$
E_{i-j}^{\varepsilon}=\left\{x \in \Omega:\left|u^{\varepsilon}(x)\right| \geq i-j\right\} .
$$

Therefore,

$$
\begin{gathered}
\frac{1}{2} \int_{\Omega}\left|\nabla\left(u^{\varepsilon}-u\right)\right|^{2} \leq \frac{1}{2} \int_{F_{i, j}^{\varepsilon}}\left|\nabla\left(u^{\varepsilon}-u\right)\right|^{2}+\frac{1}{2} \int_{E_{i-j}^{\varepsilon}}\left|\nabla\left(u^{\varepsilon}-u\right)\right|^{2} \\
\leq \int_{F_{i, j}^{\varepsilon}}\left|\nabla\left(u^{\varepsilon}-T_{j}(u)\right)\right|^{2}+\int_{F_{i, j}^{\varepsilon}}\left|\nabla\left(T_{j}(u)-u\right)\right|^{2} \\
\quad+\int_{E_{i-j}^{\varepsilon}}\left|\nabla u^{\varepsilon}\right|^{2}+\int_{E_{i-j}^{\varepsilon}}|\nabla u|^{2} \leq 2\left(A_{i j}^{\varepsilon}+B_{i j}^{\varepsilon}+C_{i j}^{\varepsilon}+D_{i j}^{\varepsilon}\right) .
\end{gathered}
$$

We have seen in (19) that

$$
\lim _{j \rightarrow+\infty} \limsup _{\varepsilon \rightarrow 0} A_{i j}^{\varepsilon}=0 \quad \forall i \geq 1
$$

The second term $B_{i j}^{\varepsilon}$ satisfies

$$
\limsup _{\varepsilon \rightarrow 0} B_{i j}^{\varepsilon} \leq \int_{\Omega}\left|\nabla\left(T_{j}(u)-u\right)\right|^{2}
$$


whence we also have

$$
\lim _{j \rightarrow+\infty} \limsup _{\varepsilon \rightarrow 0} B_{i j}^{\varepsilon}=0 \quad \forall i \geq 1
$$

From (15) we know that

$$
\lim _{j \rightarrow+\infty} \limsup _{\varepsilon \rightarrow 0} C_{i j}^{\varepsilon}=0 \quad \text { as } i, j \rightarrow+\infty, i-j \rightarrow+\infty .
$$

Finally, this is also true for $D_{i j}^{\varepsilon}$, since $u \in H_{0}^{1}$ :

$$
\lim _{j \rightarrow+\infty} \limsup _{\varepsilon \rightarrow 0} D_{i j}^{\varepsilon}=0 \quad \text { as } i, j \rightarrow+\infty, i-j \rightarrow+\infty .
$$

From (25) and (26)-(29), we deduce at once that $u^{\varepsilon} \rightarrow u$ strongly in $H_{0}^{1}$ as $\varepsilon \rightarrow 0$.

Fifth step. End of the proof of theorem 1.1.

Let us chose $h \in C_{c}^{1}(\mathbb{R})$ and $\varphi, \psi \in \mathcal{D}$. Multiplying the first equation in (6) by $h\left(u^{\varepsilon}\right) \varphi$ and the second one by $\psi$ and integrating by parts, we obtain:

$$
\left\{\begin{array}{l}
\int_{\Omega}\left(\nabla u^{\varepsilon}+\beta\left(v^{\varepsilon}\right)\left(X^{\varepsilon}\right)^{\prime}\left(u^{\varepsilon}\right)\right) \cdot \nabla\left(h\left(u^{\varepsilon}\right) \varphi\right)=\left\langle f, h\left(u^{\varepsilon}\right) \varphi\right\rangle \\
\int_{\Omega}\left(\nabla v^{\varepsilon}+\beta^{\prime}\left(v^{\varepsilon}\right) X^{\varepsilon}\left(u^{\varepsilon}\right)\right) \cdot \nabla \psi=\langle g, \psi\rangle .
\end{array}\right.
$$

Since $h$ and $h^{\prime}$ have compact support on $\mathbb{R}$, for $\varepsilon$ sufficiently small we have

$$
\left(X^{\varepsilon}\right)^{\prime}(t) h(t)=X^{\prime}(t) h(t), \quad\left(X^{\varepsilon}\right)^{\prime}(t) h^{\prime}(t)=X^{\prime}(t) h^{\prime}(t) .
$$

Both functions belong to $\left(C^{0}(\mathbb{R}) \cap L^{\infty}(\mathbb{R})\right)^{N}$. Thus, we can write (30) as follows

$$
\left\{\begin{array}{l}
\int_{\Omega} h\left(u^{\varepsilon}\right) \nabla u^{\varepsilon} \cdot \nabla \varphi+\int_{\Omega} h^{\prime}\left(u^{\varepsilon}\right)\left|\nabla u^{\varepsilon}\right|^{2} \varphi+\int_{\Omega} \beta\left(v^{\varepsilon}\right) h\left(u^{\varepsilon}\right) X^{\prime}\left(u^{\varepsilon}\right) \cdot \nabla \varphi \\
+\int_{\Omega} \beta\left(v^{\varepsilon}\right) h^{\prime}\left(u^{\varepsilon}\right)\left(X^{\prime}\left(u^{\varepsilon}\right) \cdot \nabla u^{\varepsilon}\right) \varphi=\left\langle f, h\left(u^{\varepsilon}\right) \varphi\right\rangle \\
\left.\int_{\Omega} \nabla v^{\varepsilon} \nabla \psi+\int_{\Omega} \beta^{\prime}\left(v^{\varepsilon}\right) X\left(u^{\varepsilon}\right)\right) \cdot \nabla \psi=\langle g, \psi\rangle .
\end{array}\right.
$$


Now, using the strong convergence of $u^{\varepsilon}$ to $u$ in $H_{0}^{1}(\Omega)$, it is easy to pass to the limit in each term of (31); this yields

$$
\left\{\begin{aligned}
\int_{\Omega} h(u) \nabla u \cdot \nabla \varphi+\int_{\Omega} h^{\prime}(u)|\nabla u|^{2} \varphi & +\int_{\Omega} \beta(v) h(u) X^{\prime}(u) \cdot \nabla \varphi \\
\quad+\int_{\Omega} \beta(v) h^{\prime}(u)\left(X^{\prime}(u) \cdot \nabla u\right) \varphi & =\langle f, h(u) \varphi\rangle \\
\int_{\Omega} \nabla v \cdot \nabla \psi+\int_{\Omega} \beta^{\prime}(v) X(u) \cdot \nabla \psi & =\langle g, \psi\rangle .
\end{aligned}\right.
$$

This completes the proof.

Acknowledgment. The author is indebted to E. Fernández-Cara, for several fruitful discussions.

\section{References}

[1] J. Baranger, A. Mikelić, Solutions stationnaires pour un écoulement quasinewtonien avec echauffement visqueux, C. R. Acad. Sci. Paris, t. 319, Série I, p. 637-642, 1994.

[2] P. Benilan, L. Boccardo, T. Gallouët, R. Gariepy, J.L. Vázquez, On the p-Laplacian on $L^{1}$, to appear.

[3] L. Boccardo, J.I. Díaz, D. Giachetti, F. Murat, Existence of a solution for a weaker form of a nonlinear elliptic equation, In "Recent Advances in Nonlinear Elliptic and Parabolic Problems", P. Benilan et al. eds., Pitman Research Notes in Math., 208, Longman, Harlow 1989.

[4] R. DiPerna, P.L. Lions, On the Cauchy problem for Boltzmann equations: global existence and weak stability, Annals of Math. (2) 130 (1989), No. 2, p. 321-366.

[5] R. Lewandowski, Les équations de Stokes et de Navier-Stokes couplées avec l'équation de l'énergie cinétique turbulente, C. R. Acad. Sci. Paris, t. 381, Série I, p. 1097-1102, 1994.

[6] P.L. Lions, F. Murat, Solutions renormalisées d'équations elliptiques, to appear.

[7] F. Murat, Soluciones renormalizadas de EDP elipticas no lineales, Lectures at the University of Sevilla (may 1992), Research Report R93023, LAN, University Paris VI, 1993. 
Department of Differential Equations and Numerical Analysis

University of Sevilla

Tarfia s/n, E-41012 Sevilla

Spain

E-mail: blanca@numer.us.es.

Recibido: 13 de Marzo de 2000

Revisado: 13 de Diciembre de 2001 